\title{
Nuss repair of pectus excavatum after surgery for congenital heart disease: Experience from a single institution
}

\author{
Shuai Li, MD, Shao-tao Tang, MD, Qiangsong Tong, MD, Ying Yang, MD, Li Yang, MD, \\ Shiwang Li, MD, and Jiarui Pu, MD
}

\begin{abstract}
Objective: Pectus excavatum developing after surgery for congenital heart disease has its own clinical characteristics. The present study aimed to present our technique and outcomes for the Nuss procedure in the repair of these cases.
\end{abstract}

\begin{abstract}
Methods: We conducted a retrospective study of all patients who had not been diagnosed as pectus excavatum preoperatively but subsequently had developed pectus excavatum after surgery for congenital heart disease from February 2005 to November 2012. The Nuss procedure was applied using a series of perioperative management techniques. The data relating to the surgical technique, complications, and outcomes were analyzed. The clinical evaluation was performed using the Nuss criteria.
\end{abstract}

\begin{abstract}
Results: A total of 30 cases (14 boys and 16 girls) were included. The mean operative time was 73.5 minutes (range, 58-82). The mean length of hospital stay was 6.0 days. Complications occurred in 5 patients $(16.7 \%)$, including asymptomatic pneumothorax, hematoma in the wound, pericardial penetration, and bar displacement. The mean follow-up period was 32 months (range, 9-60). Initially, 29 patients $(96.7 \%)$ had excellent results, and 1 patient had a good result. The mean point of bar removal was 35.8 months (range, 30-39) after implantation. The postoperative results after bar removal in 17 patients were also recorded, including excellent results in 14 $(82.4 \%)$, good results in $2(11.7 \%)$, and a fair result in 1 patient.
\end{abstract}

Conclusions: The Nuss procedure has been shown to be a safe and effective approach for the repair of pectus excavatum after surgery for congenital heart disease, although dissection of substernal adhesions can increase the risk of heart injury. (J Thorac Cardiovasc Surg 2014;148:657-61)

Pectus excavatum can, in some cases, develop after surgery for congenital heart disease. For these patients, the thoracic deformity and the mechanical compression of the heart can cause or exacerbate hemodynamic instability and expose them to increased risks of complications from substernal adhesions. ${ }^{1}$ They all met $\geq 2$ of the criteria created by Nuss and Kelly ${ }^{2}$ and Redlinger and colleagues, ${ }^{3}$ indicating that the deformity should be corrected.

Currently, a scarcity of data is available regarding the use of the Nuss procedure for pectus excavatum after surgery for congenital heart disease. Since 2004, this procedure has been routinely applied for the correction of simple pectus excavatum in our institution. Our experience has helped to decrease the associated complications, ${ }^{4}$ and we have developed techniques to manage substernal adhesions. ${ }^{1,5-7}$ In 2011, Shu and colleagues ${ }^{8}$ reported a series of 406

\footnotetext{
From the Department of Pediatric Surgery, Union Hospital, Tongji Medical College, Huazhong University of Science and Technology, Wuhan, China.

Disclosures: Authors have nothing to disclose with regard to commercial support.

Received for publication Aug 16, 2013; revisions received Dec 16, 2013; accepted for publication Jan 10, 2014; available ahead of print Feb 12, 2014.

Address for reprints: Shao-tao Tang, MD, Department of Pediatric Surgery, Union Hospital of Tongji Medical College, Huazhong University of Science and

Technology, No. 1277 Jie Fang Ave, Wuhan 430022, China (E-mail: tshaotao83@126.com).

$0022-5223 / \$ 36.00$

Copyright (c) 2014 by The American Association for Thoracic Surgery

http://dx.doi.org/10.1016/j.jtcvs.2014.01.014
}

patients using minimally invasive Nuss repair, including 9 patients with a previous operation for congenital heart disease. However, the peculiarity of these 9 cases was not discussed.

For the past few years, we have used a modified thoracoscopic Nuss repair for the correction of the thoracic deformity in 30 children who had developed pectus excavatum after repair of congenital heart disease. This technique provided safe access to the lowest point of the sternum and satisfactory results according to the Nuss criteria.

\section{METHODS \\ Patients}

The present study was approved by the ethics committee of the Union Hospital of Tongji Medical College, Huazhong University of Science and Technology (Wuhan, China).

A total of 30 patients, who had undergone the Nuss procedure for postoperative pectus excavatum after surgery for congenital heart disease at the Union Hospital of Huazhong University of Science and Technology from February 2005 to November 2012, were included in the present retrospective study. Of the 30 patients, 14 were boys and 16 were girls, with a mean age of 5.5 years (range, 2.7-12.9). The mean Haller index (HI) determined using computed tomography was $3.8 \pm 0.6$ (range, 2.76-5.8) According to the simplified version of Park's classification, ${ }^{9} 24$ patients had a symmetric type and 6 had an unbalanced type (Figure 1). The different types of combined congenital heart disease are listed in Table 1.

The parents' consent for the operation was obtained after they had been informed of the surgical indications, complications, possible outcomes, and limitations of the Nuss procedure. 


\section{Abbreviation and Acronym}

$\mathrm{HI}=$ Haller index

The patient characteristics, operative time, length of hospital stay, efficiency of the operation, and postoperative complications were recorded.

\section{Surgical Technique}

All patients underwent thoracoscopic Nuss repair, as described previously. $^{7}$

The main steps were as follows:

Preoperative imaging evaluation of the adhesions between the sternum and pericardium (or heart)

Applying an open access technique in trocar introduction to prevent possible lung injury caused by the pleura adhesions

Assessing the degree of adhesions after inserting the thoracoscope to determine whether a subxiphoid approach ${ }^{7}$ or a modified bilateral thoracoscopic repair ${ }^{6}$ should be used

To prevent flaring of the costal arch after Nuss repair, after the introducer has been placed in the proper position inside the thoracic cavity, upward traction on the introducer should be followed by downward pressure on the costal arch to cause a reduction in the deformity; this maneuver was usually repeated (approximately 10 times) until the desired new structural shape of the chest had been obtained

Postoperatively, gauze pads were placed on the peak of the rib arch bilaterally under the bandage, bound by elastic bandage, and untied 1 week later to prevent flaring

Before closing the pleural cavity, $30 \mathrm{~cm} \mathrm{H}_{2} \mathrm{O}$ of positive end-expiratory pressure was applied 8 times by hand to prevent pleural air trapping. The last session of positive end-expiratory pressure was held for 5 seconds, during which the incision was closed completely

\section{Follow-up}

The patients were followed up postoperatively at 1, 3, 12, and 24 months. After bar removal, they were followed up once a month for the next 6 months and then annually. The follow-up assessments included physical examination, chest computed tomography scan, and cardiac ultrasonography.

According to the Nuss criteria, ${ }^{3}$ the outcome of the operation can be divided into 4 grades: excellent, if the preoperative symptoms were resolved and the chest appearance was normal; good, if the preoperative symptoms were resolved and the chest appearance was improved; fair, if the preoperative symptoms were improved but the chest appearance was not completely normal; and poor, if the preoperative symptoms were not improved and the chest appearance was not completely normal.

\section{Statistical Analysis}

The descriptive characteristics are presented as the mean \pm standard deviation. Data analysis was performed using the Statistical Package for Social Sciences, version 18.0 (SPSS Inc, Chicago, Ill).

\section{RESULTS}

A total of 30 patients with pectus excavatum detected after surgery for congenital heart disease underwent the Nuss procedure. A retrospective analysis was undertaken to summarize the intraoperative and postoperative complications, the initial outcomes of the Nuss repair, and the results after bar removal. The mean follow-up period was 32 months (range, 9-60). The mean operative time was $73.5 \pm 4.7 \mathrm{mi}-$ nutes (range, 58-82). The mean length of hospital stay was $6.0 \pm 1.4$ days (range, $5-8$; Table 2 ). The mean interval to the detection of pectus excavatum was 3.1 months (range, 1.2-10) after heart surgery. The mean interval to bar removal was 35.8 months (range, 30-39) after the Nuss procedure (Table 2), and mean follow-up period for these patients was 43 months (range, 35-60).

\section{Pain Management}

Intravenous pethidine or tramadol was used for 1 to 2 days after the operation, followed by oral diclofenac sodium for about 1 week.

\section{Antibiotics}

The patients were given perioperative antibiotics to prevent postoperative infections. All the patients received antibiotic treatment for 7 days, except for 1, who had received antibiotics for 3 more days, because of a greater risk of infection owing to the development of a hematoma in the wound.

\section{Complications}

Complications occurred in 5 patients $(16.7 \%)$, with no deaths or cardiac perforations. The early postoperative complications included asymptomatic pneumothorax in 2 patients. None of the patients required a chest tube, and none developed hemothorax. One of the patients experienced pericardial penetration during the operation. The introducer was withdrawn, dissection was continued, the sternum was pulled outside the thoracic cavity with the help of towel forceps, and the introducer was advanced across the mediastinum successfully. Afterward, the patient was observed for 10 minutes, during which no active bleeding or any abnormality on the electrocardiogram was observed, and a pericardial patch was not required. Another patient experienced hematoma in the wound, but no infection occurred. One of the patients encountered mild bar displacement after falling from his bicycle, but no corrective action was required (Table 2).

\section{Outcomes}

According to the Nuss criteria, postoperative treatment of pectus excavatum after surgery for congenital heart disease using the Nuss procedure provided encouraging results. The initial observation revealed that 29 patients $(96.7 \%$ ) had had excellent results, and 1 patient had achieved a good outcome. None of the patients had a fair or poor outcome. The postoperative assessments after bar removal were also performed for 17 patients, and the results were excellent in 14 patients $(82.4 \%)$, good in $2(11.7 \%)$, and fair in $1(5.9 \%)$, with no poor result in any patient (Table 2$)$. 


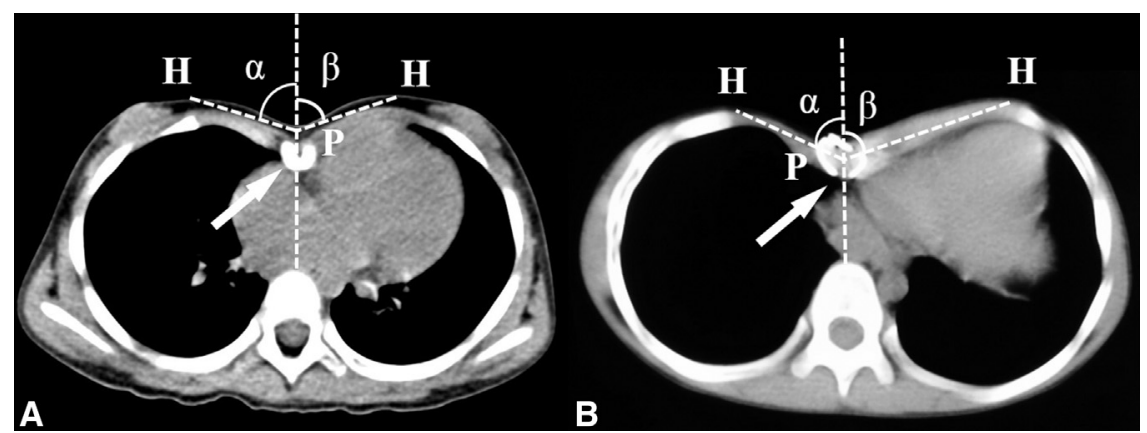

FIGURE 1. Two types of pectus excavatum after surgery for congenital heart disease. $H$, Peak of the costal arch; $P$, deepest portion of the pectus defect; $\alpha$, $\beta$, angle between the PH line and the midline line. Arrow indicates sternal fixation wire under operation for congenital heart disease. A, Symmetric pectus excavatum, $\alpha=\beta$. B. Unbalanced pectus excavatum, $\alpha \neq \beta$.

\section{DISCUSSION}

Pectus excavatum can be associated with congenital heart disease. ${ }^{10}$ However, we observed some cases of pectus excavatum developing soon after surgery to repair congenital heart disease. The incidence of pectus deformity developing after surgery for congenital heart disease has been approximately $0.53 \%$ in our institution.

This type of pectus excavatum can be characterized as symmetric, asymmetric, or unbalanced, according to the simplified Park classification criteria. ${ }^{9}$ However, most cases $(80 \%)$ have been symmetric. In the present study, 1 patient had mild, 15 patients had moderate (HI, $>3.2$ but $\leq 3.5$ ), 14 patients had severe (HI, $\geq 3.6$ but $<6.0$ ), and none of the patients had extremely severe $(\mathrm{HI}, \geq 3.6$ but $<6.0)$ pectus

\section{TABLE 1. Clinical data}

\begin{tabular}{lc}
\hline \multicolumn{1}{c}{ Variable } & Value \\
\hline Gender & \\
Male & 14 \\
Female & 16 \\
Age $(\mathrm{y})$ & \\
Mean & 5.5 \\
Range & $2.7-12.9$ \\
HI & \\
Mean & 3.8 \\
Range & $2.76-5.8$ \\
Type & \\
Symmetric & 24 \\
Asymmetric & 0 \\
Unbalanced & 6 \\
Associated malformations & \\
VSD & 12 \\
Atrial septal defect & 18 \\
VSD and ASD & 1 \\
Patent ductus arteriosus & 6 \\
Aortic stenosis & 1 \\
Tetralogy of Fallot & 2 \\
Scoliosis & 3 \\
Flaring of the costal arch & 3 \\
\hline$H I$ Haller index; $V S D$, ventricular septal defect; $A S D$, atrial septal defect. \\
\end{tabular}

excavatum. Substernal adhesions could be seen in all cases, but most could be successfully separated. Dissecting the substernal adhesions can be the main cause of a prolonged operation time. The average point at which the chest wall abnormality was detected was 3.1 months after congenital heart disease repair. More attention should be given to the possibility of pectus excavatum developing after surgery for congenital heart disease.

The etiology and mechanism of pectus excavatum have not been fully elucidated. Many studies have described the immature pattern of the cartilage in patients with pectus excavatum. $^{11}$ A proposed mechanism has been an abnormal pulling force of the sternum to the diaphragm posteriorly, ${ }^{12}$ revealing the important role of unbalanced

TABLE 2. Complications and results

\begin{tabular}{lc}
\hline \multicolumn{1}{c}{ Variable } & Results \\
\hline Mean blood loss (mL) & 5 \\
Operative time (min) & \\
Mean & 73.5 \\
Range & $58-82$ \\
Length of hospital stay (d) & \\
Mean & 6.0 \\
Range & $5-8$ \\
Drainage tube placement & 0 \\
Postoperative complications & $5(16.7)$ \\
Pneumothorax & $2(6.7)$ \\
Hemothorax & 0 \\
Hematoma in wound & $1(3.3)$ \\
Pericardial penetration & $1(3.3)$ \\
Cardiac perforation & 0 \\
Bar displacement & 1 \\
Initial results (n = 30) & \\
Excellent & $29(96.7)$ \\
Good & $1(3.3)$ \\
Fair & $0(0)$ \\
Results after bar removal (n = 17) & \\
Excellent & $14(82.4)$ \\
Good & $2(11.7)$ \\
Fair & $1(5.9)$ \\
\hline Data presented as n (\%), unless noted otherwise. &
\end{tabular}


forces in the process. Several features will be inconsistent with congenital pectus excavatum. First, the average point at which the chest wall abnormality became obvious was 3.1 months postoperatively in patients ranging in age from 2.7 to 12.9 years. In contrast, most cases $(86 \%)$ of congenital pectus excavatum will be noted in the first year of life. ${ }^{12}$ Thus, our cases were not consistent with the natural progression of congenital pectus excavatum. Second, we had 14 boys and 16 girls in our series; however, congenital pectus excavatum occurs much more frequently (with a 5:1 ratio) in boys than in girls. ${ }^{13}$ Furthermore, all our patients had undergone a sternotomy during their heart operation, and this might have caused unbalanced stress on the sternum and the development of an immature pattern of cartilage in the sternum. Therefore, it has been hypothesized that the combination of unbalanced forces inside the chest and an immature pattern of composition might be responsible for pectus excavatum developing soon after surgery for congenital heart disease. Once the unbalanced forces inside the chest were corrected by the Nuss operation, the normal appearance of the chest was restored. However, more studies, using a large number of patients, are needed to confirm the correlation between the development of pectus excavatum and surgery for congenital heart disease.

The Nuss criteria were applied to determine the indications for these patients. ${ }^{2}$ Although the surgical indications have been extended from children to adults ${ }^{14,15}$ and from symmetric to asymmetric, ${ }^{16}$ we had 3 patients $(10 \%)$ who were $\leq 4$ year old. Two of them demonstrated rapid progression in pectus malformation. Another patient presented with decreased exercise tolerance 3 months after heart surgery and was the only patient with a $\mathrm{HI}<3.25$ (HI, 2.76).

Generally, patients with pectus excavatum after surgery for congenital heart disease can safely undergo the Nuss procedure with minimal complications. The overall incidence of complications was around $16.7 \%$, similar to that in the study by Kelly and colleagues. ${ }^{17}$ The key point is to dissect the retrosternal adhesion. The right atrial appendage or pericardium can be ripped by hasty dissection, with fatal risks. To decrease the risk of heart injury, mediastinal dissection by the introducer should be performed using direct thoracoscopic visualization and as close to the sternum as possible. If necessary, the sternum should be pulled or pressed using towel forceps in vitro, combined with dissection in the thoracic cavity, which can increase, not only the operational accuracy, but also the tactile feedback. A modified bilateral thoracoscopic repair can be helpful ${ }^{6}$; however, we have not yet tried it. The routine use of a subxiphoid approach has been previously described, during which the surgeon's fingers can be introduced to manually guide the introducer tip beneath the sternum in lieu of thoracoscopy. ${ }^{7}$ It would be much easier and safer if effective adhesion prevention measures could be taken during the heart surgery. Although adhesion preventing membranes have been used routinely, the pericardium has not been routinely closed during the heart operation. Therefore, little benefit has been derived from the use of the membrane. More effective adhesion prevention measures need to be explored.

Although correction of pectus excavatum after surgery for congenital heart disease can be difficult and complex, the postoperative results have been satisfactory. A recent meta-analysis showed that $93.3 \%$ of simple cases of the Nuss procedure achieved good to excellent results. ${ }^{18}$ In the present series, $82.4 \%$ of patients achieved excellent results and $11.7 \%$ patients, good results, similar to the results from the meta-analysis. The results of 3 patients had deteriorated after bar removal, including from excellent to good in 2 patients and from good to fair in 1 patient. All 3 patients were $>9$ years and had had the bars removed $<3$ years after the Nuss procedure. The deterioration was detected weeks after bar removal using computed tomography. After noticing this phenomenon, we provided constant follow-up ( $\geq 12$ months) and found no signs of worsening. This phenomenon had also been reported by Croitoru and colleagues ${ }^{5}$ but was not discussed in detail. We speculated that this rebound resulted from the skeletal elasticity of older patients (age, $>9$ years) and the shorter duration of bar placement. Nuss and Kelly ${ }^{3}$ reported that if the bar has been removed before 2 years postoperatively, the recurrence rate will increase inversely with the period the bar remained in situ. After 2010, all our patients had their bars removed $\geq 3$ years after the Nuss procedure, and no deterioration was found. More attention should be given to this phenomenon.

A recent study has demonstrated that Nuss repair of pectus excavatum using a novel morphology tailored, patient-specific approach is effective for quality repair of the full spectrum of pectus excavatum, including complex cases. ${ }^{19}$ However, for patients with severe deformities or those who do not wish to have the substernal bar in situ for 3 years, open repair (modified Ravitch technique) can be an alternative. However, a risk exists of impaired thoracic growth and possible restrictive pulmonary impairment after surgery owing to injury to the growth centers of the ribs and sternum. ${ }^{20}$

\section{CONCLUSIONS}

Pectus excavatum can occur after surgery for congenital heart disease. Its pathogenesis is not yet known; however, the Nuss procedure is an effective treatment of this type of pectus excavatum, although dissection of substernal adhesions increases the risk of heart injury. Special characteristics and operative techniques should be considered to decrease the complications associated with this procedure. 


\section{References}

1. Rousse N, Juthier F, Prat A, Wurtz A. Staged repair of pectus excavatum during an aortic valve-sparing operation. J Thorac Cardiovasc Surg. 2011;141:e28-30.

2. Nuss D, Kelly RE Jr. Indications and technique of Nuss procedure for pectus excavatum. Thorac Surg Clin. 2010;20:583-97.

3. Redlinger RE Jr, Kelly RE Jr, Nuss D, Kuhn MA, Obermeyer RJ, Goretsky MJ. One hundred patients with recurrent pectus excavatum repaired via the minimally invasive Nuss technique-effective in most regardless of initial operative approach. J Pediatr Surg. 2011;46:1177-81.

4. Mao YZ, Tang ST, Wang Y, Tong QS, Ruan QL. Nuss operation for pectus excavatum: a single-institution experience. World J Pediatr. 2009;5:292-5.

5. Croitoru DP, Kelly RE Jr, Goretsky MJ, Gustin T, Keever R, Nuss D. The minimally invasive Nuss technique for recurrent or failed pectus excavatum repair in 50 patients. J Pediatr Surg. 2005;40:181-6; discussion 186-7.

6. Cheng YL, Lee SC, Huang TW, Wu CT. Efficacy and safety of modified bilateral thoracoscopy-assisted Nuss procedure in adult patients with pectus excavatum. Eur J Cardiothorac Surg. 2008;34:1057-61.

7. St Peter SD, Sharp SW, Ostlie DJ, Snyder CL, Holcomb GW, Sharp RJ. Use of a subxiphoid incision for pectus bar placement in the repair of pectus excavatum. J Pediatr Surg. 2010;45:1361-4.

8. Shu Q, Shi Z, Xu WZ, Li JH, Zhang ZW, Lin R, et al. Experience in minimally invasive Nuss operation for 406 children with pectus excavatum. World J Pediatr. 2011;7:257-61.

9. Zeng Q, Zhang N, Chen CH, He YR. Classification of the pectus excavatum and minimally invasive Nuss procedure. Zhonghua Wai Ke Za Zhi. 2008;46:1160-2.

10. Shamberger RC, Welch KJ, Castaneda AR, Keane JF, Fyler DC. Anterior chest wall deformities and congenital heart disease. J Thorac Cardiovasc Surg. 1988;96:427-32.

11. David VL, Izvernariu DA, Popoiu CM, Puiu M, Boia ES. Morphologic, morphometrical and histochemical proprieties of the costal cartilage in children with pectus excavatum. Rom J Morphol Embryol. 2011;52:625-9.

12. Nobuhara KK, Lund DP, Mitchell J, Kharasch V, Wilson JM. Long-term outlook for survivors of congenital diaphragmatic hernia. Clin Perinatol. 1996;23:873-87.

13. Obermeyer RJ, Goretsky MJ. Chest wall deformities in pediatric surgery. Surg Clin North Am. 2012;92:669-84.

14. Nagasao T, Miyamoto J, Tamaki T, Ichihara K, Jiang H, Taguchi T, et al. Stres distribution on the thorax after the Nuss procedure for pectus excavatum results in different patterns between adult and child patients. J Thorac Cardiovasc Surg. 2007; 134:1502-7.

15. Schalamon J, Pokall S, Windhaber J, Hoellwarth ME. Minimally invasive correction of pectus excavatum in adult patients. J Thorac Cardiovasc Surg. 2006;132:524-9.

16. Nagasao T, Noguchi M, Miyamoto J, Jiang H, Ding W, Shimizu Y, et al Dynamic effects of the Nuss procedure on the spine in asymmetric pectus excavatum. J Thorac Cardiovasc Surg. 2010;140:1294-9.

17. Kelly RE, Goretsky MJ, Obermeyer R, Kuhn MA, Redlinger R, Haney TS, et al Twenty-one years of experience with minimally invasive repair of pectus excavatum by the Nuss procedure in 1215 patients. Ann Surg. 2010;252:1072-81.

18. Nasr A, Fecteau A, Wales PW. Comparison of the Nuss and the Ravitch procedure for pectus excavatum repair: a meta-analysis. J Pediatr Surg. 2010;45:880-6.

19. Park HJ, Jeong JY, Jo WM, Shin JS, Lee IS, Kim KT, et al. Minimally invasive repair of pectus excavatum: a novel morphology-tailored, patient-specific approach. J Thorac Cardiovasc Surg. 2010;139:379-86.

20. Chen CH, Liu HC, Hung TT, Chen CH. Restrictive chest wall deformity as a complication of surgical repair for pectus excavatum. Ann Thorac Surg. 2010 89:599-601. 